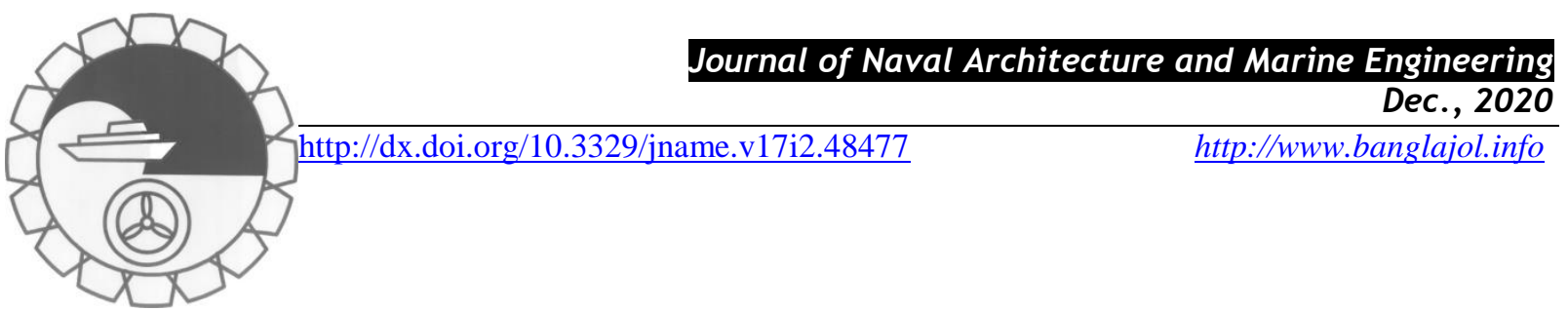

\title{
INVESTIGATION OF VARIABLE VISCOSITY AND THERMAL CONDUCTIVITY ON MHD MASS TRANSFER FLOW PROBLEM OVER A MOVING NON-ISOTHERMAL VERTICAL PLATE
}

\section{Sahin Ahmed $^{1 *}$, G. C. Hazarika ${ }^{2}$ and Geeti Gogoi ${ }^{3}$}

${ }^{1 *}$ Fluid Mechanics Research, Department of Mathematics, Rajiv Gandhi University, Rono Hills - 791112, Arunachal Pradesh, India, Email: sahin.ahmed@rgu.ac.in

${ }^{2}$ Department of Mathematics, Dibrugarh University, Dibrugarh - 786004, Assam, India, Email: gchazarika@ gmail.com

${ }^{3}$ Department of Mathematics, Dibrugarh University, Dibrugarh - 786004, Assam, India, Email: geetigogoi94@ gmail.com

\begin{abstract}
:
In this paper we investigate numerically the influence of variable viscosity and thermal conductivity on MHD convective flow of heat and mass transfer problem over a moving non-isothermal vertical plate. The viscosity of the fluid and thermal conductivity are presumed to be the inverse linear functions of temperature. With the help of similarity substitution, the flow governing equations and boundary conditions are transformed into ordinary differential equations. The boundary value problem so obtained is then solved using MATLAB bvp4c solver. The effects of various parameters viz. magnetic parameter, viscosity parameter, thermal conductivity parameter, stratification parameter and Schmidt number on velocity, temperature and concentration are obtained numerically and presented trough graphs. Also the coefficient of skin-friction, Nusselt number and Sherwood number are computed and displayed in tabular form. The effects of the viscosity parameter and thermal conductivity parameter in particular are prominent. This study has applications in a number of technological processes such as metal and polymer extrusion.
\end{abstract}

Keywords: MHD flow, variable viscosity, thermal conductivity, heat and mass transfer, convection

\begin{tabular}{|c|c|c|c|}
\hline \multicolumn{2}{|c|}{ NOMENCLATURE } & Sc & Schmidt number \\
\hline$u, v$ & velocity components along $\mathrm{x}$ and $\mathrm{y}$ axis & $D u$ & Dufour number \\
\hline$g$ & Acceleration due to gravity & $S r$ & Soret number \\
\hline$T_{m}$ & mean fluid temperature & $S h$ & $\begin{array}{l}\text { Rate of mass transfer in terms of } \\
\text { Sherwood number at } y=0\end{array}$ \\
\hline$C_{S}$ & concentration susceptibility & $B_{0}$ & Magnetic field strength \\
\hline$K_{T}$ & thermal diffusion ratio & $q$ & Dimensional heat generation \\
\hline$C$ & concentration & $\begin{array}{c}a, b, T_{c} \\
T_{r}\end{array}$ & constants \\
\hline$T$ & Temperature & & Greek symbols \\
\hline$C_{p}$ & specific heat at constant pressure & $\boldsymbol{\theta}_{r}$ & dimensionless viscosity \\
\hline$D_{m}$ & mass diffusivity & $\boldsymbol{\theta}_{c}$ & dimensionless thermal conductivity \\
\hline$C_{w}$ & species concentration & $v$ & kinematic viscosity \\
\hline$G r$ & Grashof number for heat transfer & $\beta_{\mathrm{T}}$ & thermal spreading coefficient \\
\hline$G c$ & Grashof number for mass transfer & $\beta_{\mathrm{C}}$ & concentration spreading coefficient \\
\hline$M$ & Hartmann number & $\kappa$ & thermal conductivity \\
\hline $\operatorname{Pr}$ & Prandtl number & $\sigma$ & electrical conductivity of the fluid \\
\hline$S$ & Thermal stratification parameter & $\lambda$ & thermal conductivity \\
\hline$Q$ & heat generation parameter & $\mu$ & dynamic viscosity \\
\hline
\end{tabular}




$\begin{array}{ll}\boldsymbol{U} & \text { free stream velocity } \\ T_{w} & \text { Temperature near the surface } \mathrm{y}=0 \\ T_{\infty} & \text { Temperature far away the surface } \mathrm{y}=0 \\ T_{0} & \text { Temperature at the surface } \mathrm{y}=0 \\ f^{\prime} & \text { Non-dimensional velocity } \\ C_{f} & \text { Skin friction } \\ N u & \begin{array}{l}\text { Rate of heat transfer in terms of Nusselt } \\ \text { number at } y=0\end{array}\end{array}$

$\begin{array}{cl}\mu_{\infty} & \text { dynamic viscosity of the ambient fluid } \\ v_{\infty} & \text { kinematic viscosity of the ambient fluid } \\ \theta & \text { dimensionless temperature } \\ \rho & \text { density of fluid } \\ \phi & \text { Non-dimensional species concentration } \\ \psi & \text { Stream function } \\ \eta & \text { Non-dimensional concentration }\end{array}$

\section{Introduction}

The analysis of MHD convection flow of heat and transfer of mass along a moving non-isothermal vertical plate in presence of heat generation/ absorption draw attention of many researchers due to various applications of the problem. The study of MHD flow is necessary for various fields namely-agriculture, engineering, petroleum industry etc. The convection heat transfer in the presence of magnetic field is very important in the field of astrophysics and geophysics. A good number of works have been carried out in this field. Cheesewright (1967) studied the natural convection heat transfer along an isothermal vertical plate. Helmy (1998) examined the unsteady laminar natural convection two-dimensional flow of an electrically conducting, incompressible fluid in presence of porous medium surrounded by an infinite plane vertical surface. In the direction of a wavy surface, Molla et al. (2004) studied the generation or absorption of heat effect on free convection. In presence of constant suction and heat source, Pop and Soundalgekar et al. (1962) observed natural convection unsteady flow moving in the direction of an infinite plate.

The influence of variable viscosity and thermal conductivity on natural convection steady flow and transfer of heat in the direction of an isothermal vertical plate in presence of heat sink are studied by Mahanti et al. (2009). Hazarika et al. (2018) examined the effects of variable viscosity and thermal conductivity on transient hydromagnetic flow over a hot vertical plate. Sarma et al. (2009) studied the influence of variable viscosity and thermal conductivity on transfer of mass and heat in presence of magnetic field in the direction of a vertical plate. The effects of variable viscosity and thermal conductivity on the MHD flow of a micropolar fluid which past through an accelerated infinite plate are studied by Thakur et al. (2015). Lai et al. (1990) studied the effects of variable viscosity on the transfer of heat in the direction of a vertical surface in presence of porous medium. Recently, Hazarika et al. (2020) studied the effect of variable viscosity and thermal conductivity on unsteady free convective flow of a micropolar fluid past a vertical cone. Kalita et al. (2016) analyzed the heat and mass transfer transfer Couette flow in different physical situations. Steady-state solutions of unsteady MHD free convective heat and mass transfer flow for Soret effect induced magnetic field and thermal diffusion investigated by Zueco et al. (2017). The twin effects of the magnetic field and heat flux on the threedimensional Couette flow is presented by Ahmed et al. (2017). Recently, Hazarika et al. [(2020), (2021)] presented channel flow of heat and mass transfer applications in CNT's and nanofluids for hydromagnetic forces and obtained significant outcomes in medical therapy. Moreover, Khedr et al. (2009), Modather et al. (2009) and Magyari et al. (2010) have investigated the effects of hydromagnetic heat generation or absorption on micropolar fluids with various configurations. Some significant effects on heat and mass transfer mixed convection flow in presence of chemical reactions for and nanofluids over different surfaces are highlighted by Takhar et al. (1999), Chamkha and Aly (2010) and Parvin et al. (2012).

The main purpose of this paper is to extend the work of Subhakar et al. (2012) to study the effects of the variable fluid viscosity and thermal conductivity. In the earlier paper, the flow properties viz. viscosity and thermal conductivity are taken as constant. But these properties may vary with the variation of temperature (Herwig and Gersten, 1986). So, for the correct calculation of the mass and heat transfer rate we take variation of these flow properties. We consider the fluid viscosity and thermal conductivity as an inverse linear function of temperature. The non-linear governing equations are reduced to ordinary differential equations with the help of similarity transformations and then solved numerically by employing MATLAB bvp4c solver method. The effects of variable fluid viscosity, variable thermal conductivity, magnetic parameter, Schmidt number and stratification parameter on fluid velocity, temperature and concentration are displayed graphically. 


\section{Mathematical Formulation}

Consider the laminar convective flow in steady state of an electrically conducting fluid, which is incompressible in the direction of a vertical plate. This plate is non-isothermal, non-conducting and moving with velocity $U$. Near the plate the fluid temperature is $T_{w}$ and far away from the plate the fluid temperature is $T_{\infty}$. The x-axis is taken in the direction of the plate and $y$-axis is along perpendicular to the plate. The fluid temperature is $T_{0}$ at $y=0$. Also $B_{0}$, the uniform magnetic field intensity is implementing along $y$-axis. We take external electric field for charge polarization and Hall effects as zero since the magnetic field is not very strong.

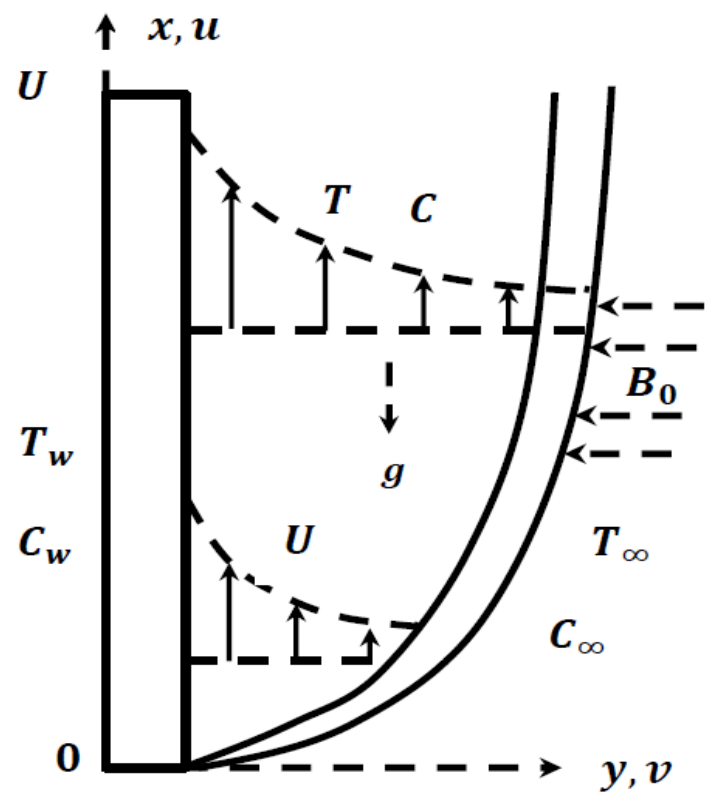

Fig. A: Schematic diagram of Flow model.

Under the Boussinesq's approximation and within the boundary layer, the governing equations are given by

\section{Continuity equation}

$$
\frac{\partial u}{\partial x}+\frac{\partial v}{\partial y}=0
$$

\section{Momentum equation}

$$
u \frac{\partial u}{\partial x}+v \frac{\partial v}{\partial y}=\frac{\partial}{\partial y}\left(v \frac{\partial u}{\partial y}\right)-\frac{\sigma B_{0}^{2}}{\rho} u+g \beta_{T}\left(T-T_{\infty}\right)+g \beta_{C}\left(C-C_{\infty}\right)
$$

\section{Energy equation}

$$
\rho C_{P}\left(u \frac{\partial T}{\partial x}+v \frac{\partial T}{\partial y}\right)=\frac{\partial}{\partial y}\left(\kappa \frac{\partial T}{\partial y}\right)+\frac{\rho D_{m} K_{T}}{C_{S}} \frac{\partial^{2} C}{\partial y^{2}}+q\left(T-T_{\infty}\right)
$$

\section{Duffusion equation}

$$
u \frac{\partial C}{\partial x}+v \frac{\partial C}{\partial y}=D_{m} \frac{\partial^{2} C}{\partial y^{2}}+\frac{D_{m} K_{T}}{T_{m}} \frac{\partial^{2} T}{\partial y^{2}}
$$

Lai and Kulacki (1990) assumed that viscosity is an inverse linear function of temperature, i.e

Or

$$
\frac{1}{\mu}=\frac{1}{\mu_{\infty}}\left[1+\delta\left(T-T_{\infty}\right)\right]
$$




$$
\frac{1}{\mu}=a\left(T-T_{c}\right), \text { where } a=\frac{\delta}{\mu_{\infty}} \quad \text { and } \quad T_{c}=T_{\infty}-\frac{1}{\delta}
$$

Following Lai and Kulaski (1990), Hazarika et al. (2020) assumed the thermal conductivity as

$$
\frac{1}{\lambda}=\frac{1}{\lambda_{\infty}}\left[1+\xi\left(T-T_{\infty}\right)\right]
$$

Or

$$
\frac{1}{\lambda}=b\left(T-T_{r}\right), \text { where } b=\frac{\xi}{\lambda_{\infty}} \quad \text { and } \quad T_{r}=T_{\infty}-\frac{1}{\xi},
$$

$T_{\infty}$ is the temperature of the free stream, $\lambda$ and $\lambda_{\infty}$ are the thermal conductivities at temperatures $T$ and $T_{\infty}$ respectively; $a, b, T_{c}$ and $T_{r}$ are constants.

The boundary conditions are

$$
\left\{\begin{array}{c}
u=U, v=0, T=T_{w}=T_{0}+b x, C=C_{w} \text { at } y=0 \\
u \rightarrow 0, T \rightarrow T_{\infty}=T_{0}+a x, C \rightarrow C_{\infty} \text { as } y \rightarrow \infty
\end{array}\right\}
$$

The equation of mass concentration (1) is satisfied identically by the Cauchy-Riemann equations

$$
u=\frac{\partial \psi}{\partial y}, v=-\frac{\partial \psi}{\partial x}
$$

where $\psi(x, y)$ is the stream function.

Let us introduce the following dimensionless variables and similarity transformations.

$$
\left\{\begin{array}{c}
M=\frac{\sigma B_{0}^{2} x^{2}}{\mu R e}, G r=\frac{g \beta_{T}\left(T_{w}-T_{\infty}\right) x}{U^{2}}, G r_{m}=\frac{g \beta_{C}\left(C_{w}-C_{\infty}\right) x}{U^{2}}, \eta=y \sqrt{\frac{U_{0}}{v x}}, \\
D u=\frac{D_{m} K_{T}\left(C_{w}-C_{\infty}\right)}{v C_{S} C_{P}\left(T_{w}-T_{\infty}\right)}, S_{r}=\frac{D_{m} K_{T}\left(T_{w}-T_{\infty}\right)}{v C_{S} C_{P}\left(C_{w}-C_{\infty}\right)}, S=\frac{a}{b}<1, v_{\infty}=\frac{\mu_{\infty}}{\rho}, \\
P r=\frac{\mu C_{P}}{\lambda}, S c=\frac{v_{\infty}}{D_{m}}, R e=\frac{U x}{v}, Q=\frac{q x}{U_{0}}, \theta(\eta)=\frac{T-T_{\infty}}{T_{w}-T_{\infty}}, \\
\phi(\eta)=\frac{C-C_{\infty}}{C_{w}-C_{\infty}}, \psi=\sqrt{v x U_{0}} f(\eta), \theta_{r}=\frac{T-T_{r}}{T_{w}-T_{\infty}}, \theta_{c}=\frac{T-T_{c}}{T_{w}-T_{\infty}}
\end{array}\right\}
$$

Using the transformations (5) and (6), eqs. (2) to (4) reduced to

$$
\begin{aligned}
& \frac{\theta_{r}}{\left(\theta-\theta_{r}\right)^{2}} f^{\prime \prime} \theta^{\prime}-\frac{\theta_{r}}{\theta-\theta_{r}} f^{\prime \prime \prime}-M f^{\prime}+G r \theta+G c \phi+\frac{1}{2} f f^{\prime \prime}=0, \\
& -\frac{\theta_{c}}{\theta-\theta_{c}} \theta^{\prime \prime}+\frac{\theta_{c}}{\left(\theta-\theta_{c}\right)^{2}}\left(\theta^{\prime}\right)^{2}+\operatorname{Pr} D u \phi^{\prime \prime}+\operatorname{Pr} Q \theta+\frac{1}{2} \operatorname{Pr} f \theta^{\prime}=0, \\
& \phi^{\prime \prime}+\frac{1}{2} S c f \phi^{\prime}+S_{r} S c \theta^{\prime \prime}=0,
\end{aligned}
$$

The boundary conditions $(7)$ reduced to

$$
\left\{\begin{array}{l}
f(0)=0, f^{\prime}(0)=1, \quad \theta(0)=1-S, \phi(0)=1 \\
f^{\prime}(\eta) \rightarrow 0, \theta(\eta) \rightarrow 0, \quad \phi(\eta) \rightarrow 0 \text { as } \eta \rightarrow \infty
\end{array}\right\}
$$

\section{Physical Parameters}

The important physical quantities related to this problem are skin friction coefficient $\left(C_{f}\right)$, Nusselt number $(\mathrm{Nu})$ and Sherwood number $(S h)$ which are discussed in the section 3.1, 3.2 and 3.3.

\subsection{Coefficient of skin- friction, $C_{f}$}

Physically the coefficient of skin friction means the shear stress at the wall. The coefficient of skin friction on the plate $\mathrm{y}=0$ is given by

Using the transformations (9) we get

$$
C_{f}=\frac{2 \tau_{w}}{\rho \mu_{\infty}^{2}}, \quad \tau_{w}=\left.\mu \frac{\partial u}{\partial y}\right|_{y=0}, \text { where } \tau_{w} \text { is the shear stress at } y=0 .
$$

$$
C_{f}=\frac{2 \theta_{r}}{1-\theta_{r}} R e^{-1 / 2} f^{\prime \prime}(0) \Rightarrow \frac{1}{2} R e^{\frac{1}{2}} C_{f}=\frac{\theta_{r}}{1-\theta_{r}} f^{\prime \prime}(0)
$$

\subsection{Nusselt number}


Physically the Nusselt number gives the rate of heat transfer from the plate to the fluid. Nusselt number is given by

$$
N u=\frac{x q_{w}}{\lambda_{\infty}\left(T_{w}-T_{\infty}\right)}, \quad q_{w}=-\left.\lambda \frac{\partial T}{\partial y}\right|_{y=0}, \text { where } q_{w} \text { is the heat flux. }
$$

Using the transformations (9), we get

$$
N u=\frac{\theta_{c}}{1-\theta_{c}} R e^{1 / 2} \theta^{\prime}(0) \Rightarrow R e^{-1 / 2} N u=\frac{\theta_{c}}{1-\theta_{c}} \theta^{\prime}(0)
$$

\subsection{Sherwood number}

Physically the Sherwood number gives the rate of mass transfer from the plate $y=0$ to the fluid. Sherwood number is given by

$$
S h=\frac{S c M_{w} x}{v_{\infty}\left(C_{w}-C_{\infty}\right)}, \quad M_{w}=-\left.D \frac{\partial C}{\partial y}\right|_{y=0}, \text { where } M_{w} \text { is the mass flux. }
$$

Using the transformations (9), we get

$$
S h=-R e^{\frac{1}{2}} \phi^{\prime}(0) \Rightarrow R e^{-\frac{1}{2}} S h=-\phi^{\prime}(0)
$$

\section{Validity and Accuracy}

Table 1: Comparison of skin friction coefficient $C_{f}$, Sherwood number $S h$, Nusselt number $N u$ with the previous result (Subhankar et al., 2012) by taking $M=0.5, \operatorname{Pr}=0.71, S c=0.24, G r=0.1, G c=0.5$, $S=0.2, D u=S r=Q=0.1, \theta_{r}=1=\theta_{c}$ are presented in the following tables.

Table 1: Variation of magnetic body force $(M)$ on $C_{f}, N u$ and $S h$.

\begin{tabular}{|c|c|c|l|c|l|c|}
\hline $\boldsymbol{M}$ & \multicolumn{2}{|c|}{$\boldsymbol{C}_{\boldsymbol{f}}$} & \multicolumn{2}{c|}{$\boldsymbol{N u}$} & \multicolumn{2}{c|}{ Sh } \\
\cline { 2 - 7 } & $\begin{array}{l}\text { Present } \\
\text { result }\end{array}$ & $\begin{array}{l}\text { Subhankar } \\
\text { et al., 2012 }\end{array}$ & $\begin{array}{l}\text { Present } \\
\text { result }\end{array}$ & $\begin{array}{l}\text { Subhankar } \\
\text { et al., 2012 }\end{array}$ & $\begin{array}{l}\text { Present } \\
\text { result }\end{array}$ & $\begin{array}{l}\text { Subhankar } \\
\text { et al., 2012 }\end{array}$ \\
\hline 0.1 & 0.29041 & 0.2583 & 0.297541 & 0.3242 & 0.280503 & 0.2776 \\
\hline 1.0 & 0.890977 & 0.8471 & 0.235953 & 0.2434 & 0.240162 & 0.2440 \\
\hline 2.0 & 1.285466 & 0.9874 & 0.162096 & 0.1883 & 0.219650 & 0.2256 \\
\hline 3.0 & 1.586554 & 1.4865 & 0.128072 & 0.1538 & 0.209316 & 0.2155 \\
\hline
\end{tabular}

\begin{tabular}{|c|c|c|c|c|c|c|}
\hline \multirow[t]{2}{*}{$S$} & \multicolumn{2}{|c|}{$C_{f}$} & \multicolumn{2}{|c|}{$\mathrm{Nu}$} & \multicolumn{2}{|c|}{ Sh } \\
\hline & $\begin{array}{l}\text { Present } \\
\text { result }\end{array}$ & $\begin{array}{l}\text { Subhankar } \\
\text { et al., } 2012\end{array}$ & $\begin{array}{l}\text { Present } \\
\text { result }\end{array}$ & $\begin{array}{l}\text { Subhankar } \\
\text { et al., } 2012\end{array}$ & $\begin{array}{l}\text { Present } \\
\text { result }\end{array}$ & $\begin{array}{l}\text { Subhankar } \\
\text { et al., } 2012\end{array}$ \\
\hline 0.0 & 0.602352 & 0.6559 & 0.343359 & 0.3585 & 0.247867 & 0.2593 \\
\hline 0.5 & 0.584673 & 0.5729 & 0.245191 & 0.2302 & 0.237318 & 0.2403 \\
\hline 1.0 & 0.566445 & 0.5467 & 0.158104 & 0.1462 & 0.231203 & 0.2313 \\
\hline 1.5 & 0.547618 & 0.5366 & 0.100791 & 0.0866 & 0.227176 & 0.2220 \\
\hline
\end{tabular}

Table 2: Variation of Schmidt number $(S c)$ on $C_{f}, N u$ and $S h$.

\begin{tabular}{|c|c|c|c|c|c|c|}
\hline \multirow{2}{*}{$\boldsymbol{S c}$} & \multicolumn{2}{|c|}{$\boldsymbol{C}_{\boldsymbol{f}}$} & \multicolumn{2}{c|}{ Nu } & \multicolumn{2}{c|}{ Sh } \\
\cline { 2 - 7 } & $\begin{array}{l}\text { Present } \\
\text { result }\end{array}$ & $\begin{array}{l}\text { Subhankar } \\
\text { et al., 2012 }\end{array}$ & $\begin{array}{l}\text { Present } \\
\text { result }\end{array}$ & $\begin{array}{l}\text { Subhankar } \\
\text { et al., 2012 }\end{array}$ & $\begin{array}{l}\text { Present } \\
\text { result }\end{array}$ & $\begin{array}{l}\text { Subhankar } \\
\text { et al., 2012 }\end{array}$ \\
\hline 0.24 & 0.627565 & 0.6489 & 0.170738 & 0.1833 & 0.221458 & 0.2241 \\
\hline 0.62 & 0.638510 & 0.6586 & 0.158343 & 0.1715 & 0.313442 & 0.3207 \\
\hline 0.78 & 0.642583 & 0.6622 & 0.153605 & 0.1670 & 0.352272 & 0.3613 \\
\hline
\end{tabular}

Table 3: Variation of Stratification parameter $(S)$ on $C_{f}, N u$ and $S h$.

The accuracy of the present model of variable viscosity and thermal conductivity is verified by comparing the results with published results presented by (Subhankar et al., 2012). 


\section{Results and Discussion}

The equations (2.10) to (2.12) with boundary conditions (2.13) are solved by using MATLAB bvp4c solver method and results are displayed graphically for velocity, temperature and concentration fields. The values of the parameters are taken as:

$$
M=0.5, \operatorname{Pr}=0.71, S c=0.24, G r=0.1, G c=0.5, S=0.2, D u=S r=Q=0.1, \theta_{r}=-8, \theta_{c}=-6
$$

unless otherwise stated.

In thermal conductivity, the minus sign indicates that the temperature decreases in the direction of heat transport and, hence, the temperature gradient is a negative quantity. It is mentioned that the $\boldsymbol{\theta}_{\boldsymbol{c}}>0$ represents liquid and $\boldsymbol{\theta}_{\boldsymbol{c}}<0$ represents gases.

In this investigation, we have a special attention of fluid behaviour on variable viscosity $\left(\boldsymbol{\theta}_{\boldsymbol{r}}\right)$ and thermal conductivity $\left(\boldsymbol{\theta}_{\boldsymbol{c}}\right)$ with other suitable variables and their significances are mentioned herewith.

The viscosity $\left(\boldsymbol{\theta}_{\boldsymbol{r}}\right)$ in a fluid medium is the measurement of resistive force to the flow of fluid, which signifies the internal friction of a moving fluid. A fluid with large viscosity always generates more resistive forces to the motion of fluid, as its molecules produces lot of internal friction. In nature, the movement of molecules are not possible when the viscosity is too high, for which engine will work harder and burn more fuel. The other factors that affect viscosity are temperature and the shape of the molecule. Higher temperatures will correspond to higher average kinetic energies and faster moving molecules.

Thermal conductivity $\left(\boldsymbol{\theta}_{\boldsymbol{c}}\right)$ is one of the thermophysical properties. It measures the capacity of temperature exchange between heat and cold passing through a material mass. It is the intrinsic property of a material that expresses its ability to conduct heat. The difference in the temperature initiates the physical contact between the particles, creating kinetic energy and momentum. When a particle collision happens, it transfers heat energy through the material. Wherever a temperature gradient exists, a net flow of heat will result.

A stratified fluid may be defined as the fluid with density variations in the vertical direction. Air and water both are fluids and if we consider them together then they can be seen as a stratified fluid system. Density variations in the atmosphere profoundly affect the motion of water and air. Thermal stratification occurs when two types of steam with different temperatures come into contact. Their temperature difference causes the colder and heavier water to settle at the bottom of the surface while allowing the warmer and lighter water to float over the colder water.

The solutions for various values of the parameters namely magnetic parameter Hartmann number $(M)$, viscosity parameter $\left(\boldsymbol{\theta}_{\boldsymbol{r}}\right)$, thermal conductivity parameter $\left(\boldsymbol{\theta}_{\boldsymbol{c}}\right)$, stratification parameter $(S)$ and Schmidt number $(S c)$ are obtained. The outputs of the investigation are discussed through the Graphs (Fig. 1 to Fig. 12) and Tables 4 to 9.

The Figs. $1-5$ represent the velocity distributions for the variation of magnetic parameter $(M)$, thermal conductivity $\left(\boldsymbol{\theta}_{\boldsymbol{c}}\right)$, viscosity $\left(\boldsymbol{\theta}_{\boldsymbol{r}}\right)$, thermal stratification parameter $(S)$ and Schmidt number $(S c)$.

Fig. 1 shows the velocity response $(u)$ with transverse coordinate $(\eta)$ for various magnetohydrodynamic body force parameters $(M)$. In the dimensionless momentum equation (10) the magnetic effect appears as the Lorentzian linear body force term, $-M f^{\prime}$. This acts perpendicular to the plate and effectively retards the boundary layer flow along the plate length. This happens because of the application of the transverse magnetic field, which is similar to drag force that reduces the velocity of the fluid flow. This is indeed the trend observed in Fig. 1 where a strong deceleration in the flow is achieved with an increase in $M$. However, there is no case of flow reversal i.e. velocities remain positive through the boundary layer.

Fig. 2 shows that the behaviour of thermal conductivity $\left(\boldsymbol{\theta}_{\boldsymbol{c}}\right)$ on the fluid velocity. The fluid velocity and its related boundary layer increases with the increase of thermal conductivity parameter. It is due to the reason that increasing $\boldsymbol{\theta}_{\boldsymbol{c}}$ generates the heat transport to the fluid molecules towards the surface and it leads to the enhancement of the flow velocity. 


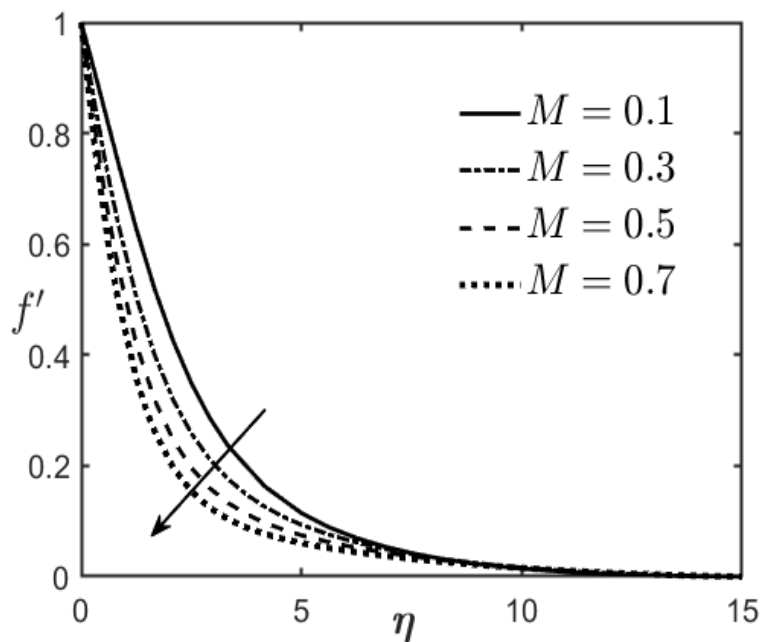

Fig. 1: Variation of velocity against $\eta$ for various values of $M$

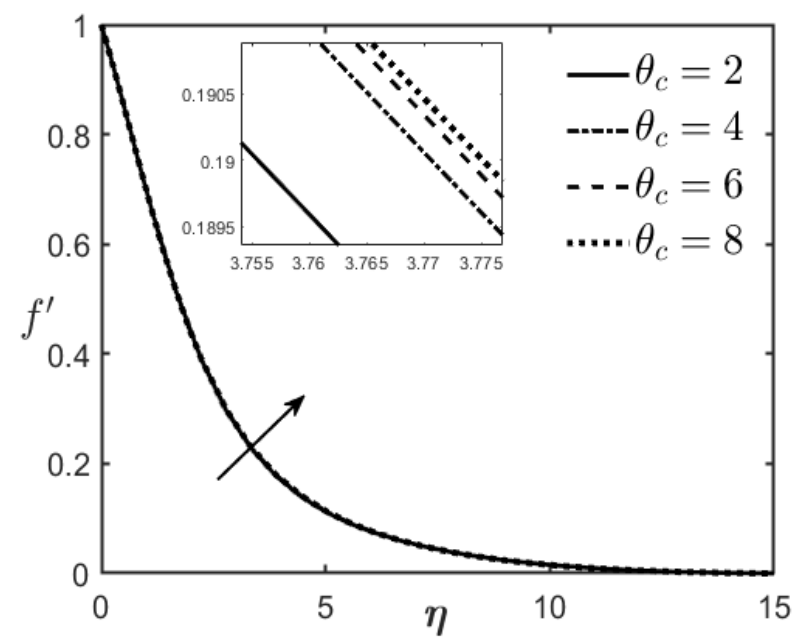

Fig. 2: Variation of velocity against $\eta$ for various values of $\boldsymbol{\theta}_{\boldsymbol{c}}$

It is observed from Fig. 3, velocity decreases with the increase of viscosity parameter $\left(\boldsymbol{\theta}_{r}\right)$. It is mentioned that the $\boldsymbol{\theta}_{\boldsymbol{r}}>0$ represents viscous forces and $\boldsymbol{\theta}_{\boldsymbol{r}}<0$ represents without viscous forces. It is due to the fact that viscous forces generates frictional forces between the fluid molecules and so the motion of the fluid becomes slow down, hence the fluid velocity finally damps within the boundary layer.

In Fig. 4, we observe that velocity decreases with the increase of thermal stratification parameter $(S)$ which is due to the decrease of the concentration gradient between the surface and the ambient. For this, the buoyancy force reduces and hence consequently declines the fluid velocity.

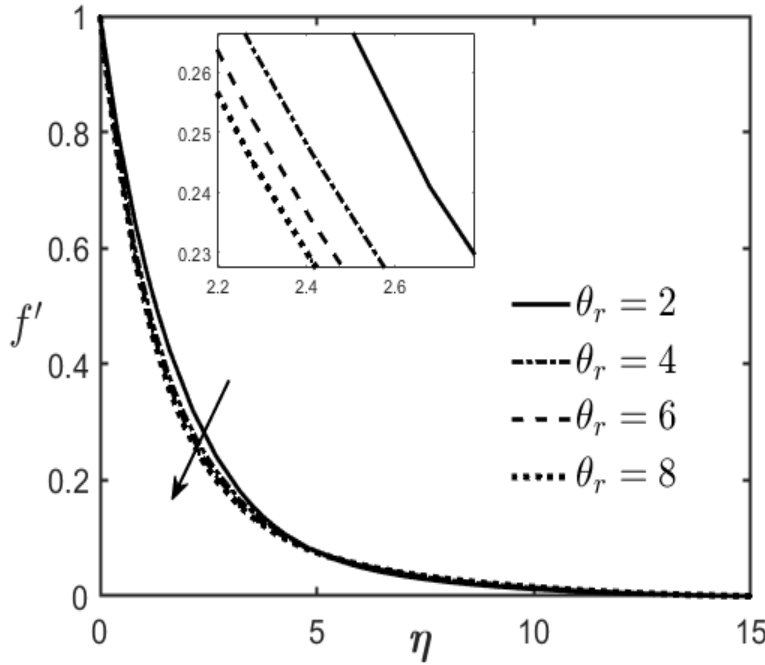

Fig. 3: Variation of velocity against $\eta$ for various values of $\boldsymbol{\theta}_{\boldsymbol{r}}$.

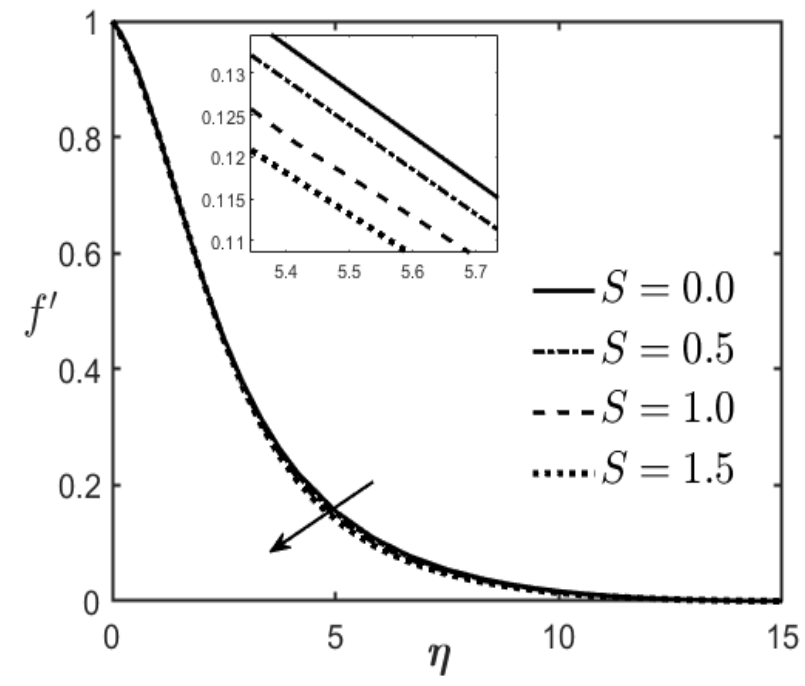

Fig. 4: Variation of velocity against $\eta$ for various values of $S$.

In Fig. 5, the impact of Schmidt number $(S c)$ on fluid velocity has illustrated. Schmidt number $(S c)$ is a dimensionless number defined as the ratio of momentum diffusivity (kinematic viscosity) and mass diffusivity, and is used to characterize fluid flows in which there are simultaneous momentum and mass diffusion convection processes. As $S c$ relates with velocity, when $S c$ increases then the molecular mass diffusivity decreases and as a result fluid flow velocity decrease. 


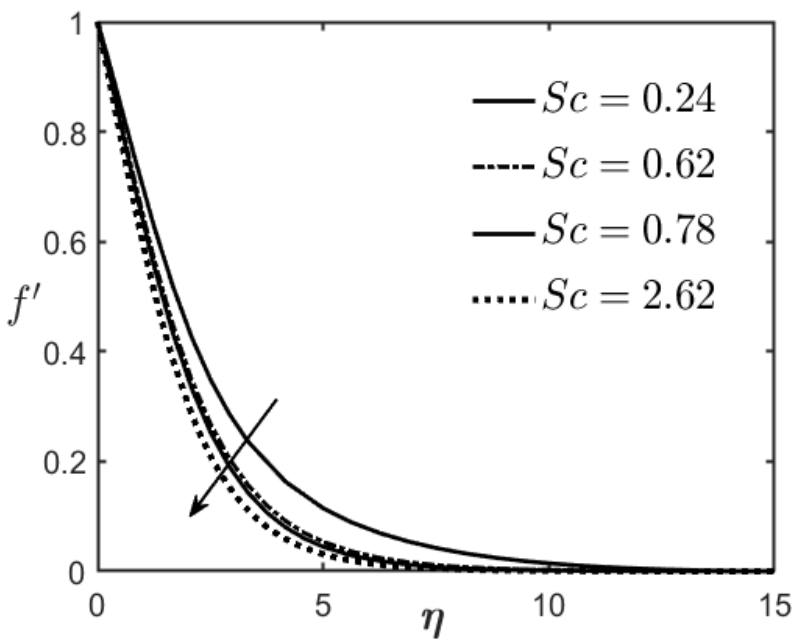

Fig. 5: Variation of velocity against $\eta$ for various values of $S c$.

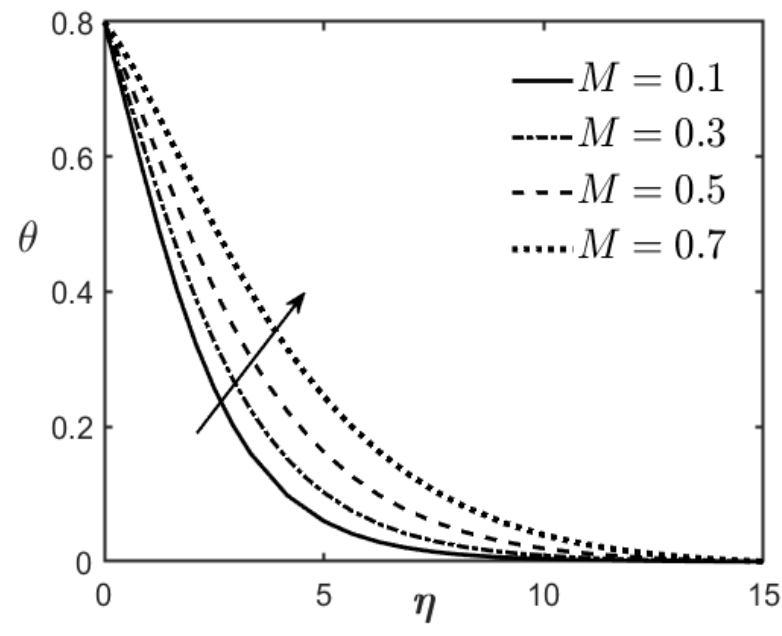

Fig. 6: Variation of temperature against $\eta$ for various values of $M$.

The temperature distributions for the physical variables magnetic parameter $(M)$, thermal conductivity $\left(\boldsymbol{\theta}_{\boldsymbol{c}}\right)$, viscosity $\left(\boldsymbol{\theta}_{\boldsymbol{r}}\right)$, thermal stratification parameter $(S)$ and Schmidt number $(S c)$ are displayed in Figs. $6-10$.

Due to the resistive force, i.e., Lorentz force the temperature enhances with the increase of magnetic parameter $(M)$ and it is shown in Fig. 6. The trend is very consistent for all $M$ values i.e. a monotonic decay from a maximum at the wall to the free stream. The supplementary work exerted by the fluid in dragging against the magnetic field retarding action, is dissipated as thermal energy, which serves to heat the fluid, and therefore, fluid temperatures are strongly enhanced with increasing magnetic field.

Variation of Temperature is illustrated in Fig. 7 for the impact of thermal conductivity parameter. The basic law of thermal conduction is the Fourier's law, which states that the heat flux density is proportional to the temperature gradient in an isotropic body. The constant of proportionality $\left(\boldsymbol{\theta}_{\boldsymbol{c}}\right)$ is the thermal conductivity. As the temperature gradient is a negative quantity, so the minus sign indicates that the temperature decreases in the direction of heat transport. This leads to the fluid temperature declined due to increase in thermal conductivity.

In Fig. 8, we observe that the temperature increases with the increase of viscosity parameter $\left(\boldsymbol{\theta}_{\boldsymbol{r}}\right)$ as heat energy increase due to the increase of viscous force. With an increase in temperature, there is typically an increase in the molecular interchange as molecules move faster in higher temperatures and therefore, with high temperatures, viscosity increases in gases $(\operatorname{Pr}=0.71)$.

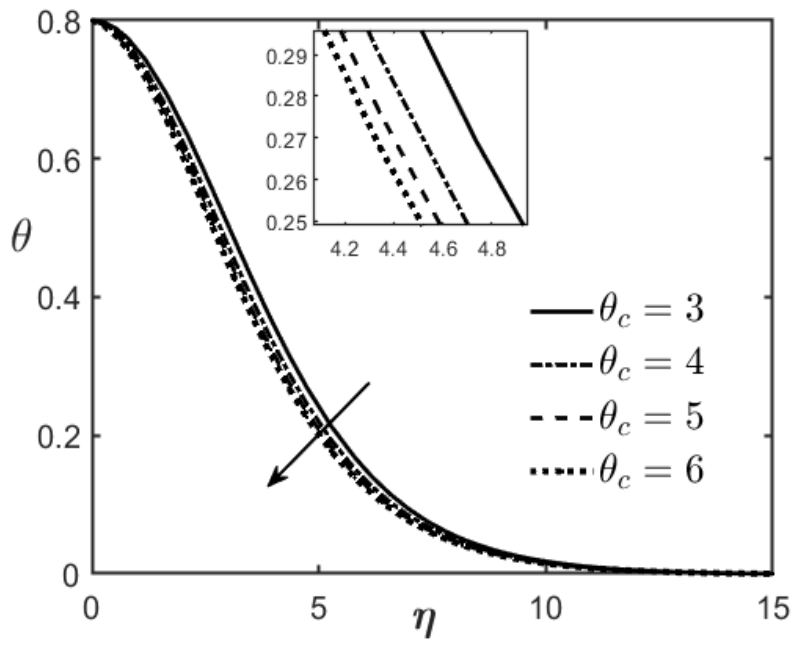

Fig. 7: Variation of Temperature against $\eta$ various values of $\boldsymbol{\theta}_{\boldsymbol{c}}$.

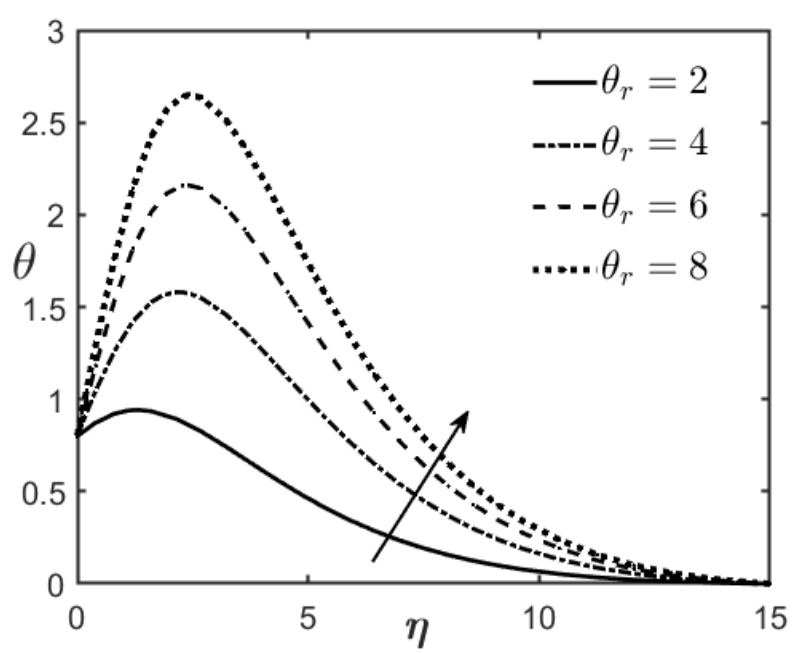

Fig. 8: Variation of Temperature against $\eta$ for various values of $\boldsymbol{\theta}_{\boldsymbol{r}}$. 


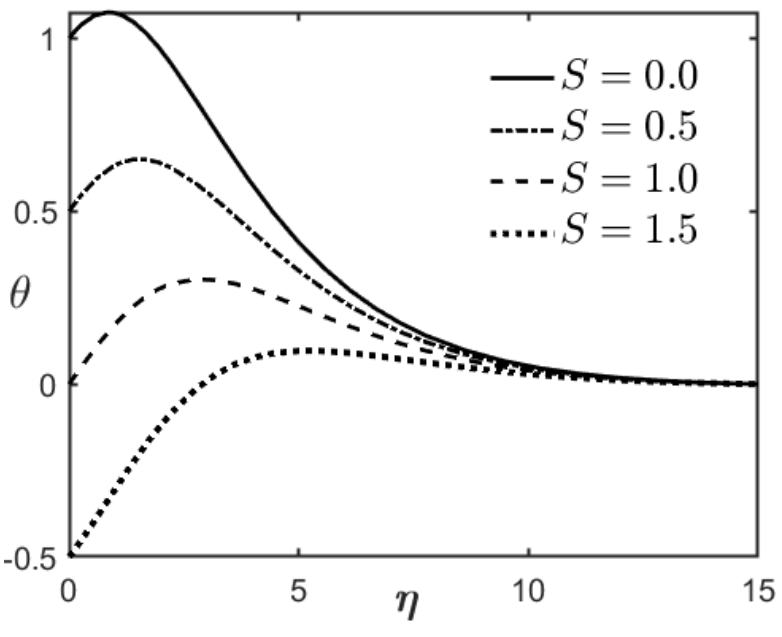

Fig. 9: Variation of Temperature against $\eta$ for various values of $S$.

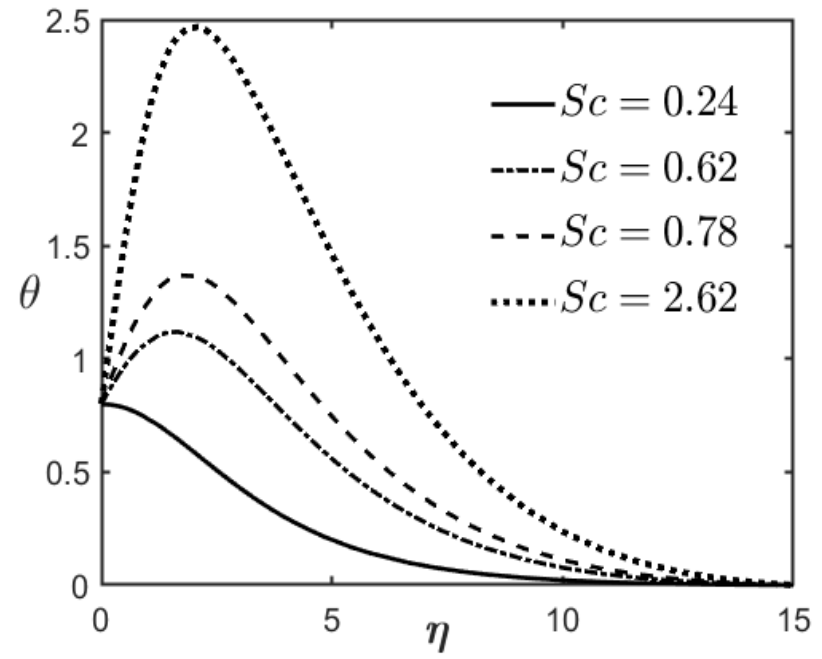

Fig. 10: Variation of Temperature against $\eta$ for various values of $S c$.

Fig. 9 is sketched for temperature field when different values of thermal stratification parameter $(S)$ are taken into account. We have seen that the temperature profile is reduced when we increase the values of thermal stratification parameter. It is also noticed that the case of prescribed surface temperature may be calculated when $\mathrm{S}=0$. Physically, the difference between the surface temperature and ambient temperature is decreased when larger values of thermal stratification parameter are used. This change in surface and ambient temperatures leads to a decrease in the temperature profile.

The effect of Schmidt number $(S c)$ on temperature of fluid is presented in Fig. 10 and this parameter is not directly involved with thermal boundary layer that means via mass transfer. The behaviour of $S c$ always reflects the opposite role of concentration of species and so it enhanced the temperature of the fluid. Moreover, the thermal boundary layer thickness increases with $S c$ because the incoming uniform flow flows over a flat plate, the fluid particles near the plate will stick to the plate (no-slip condition) that means that the momentum of the flat plate is diffused to the fluid.

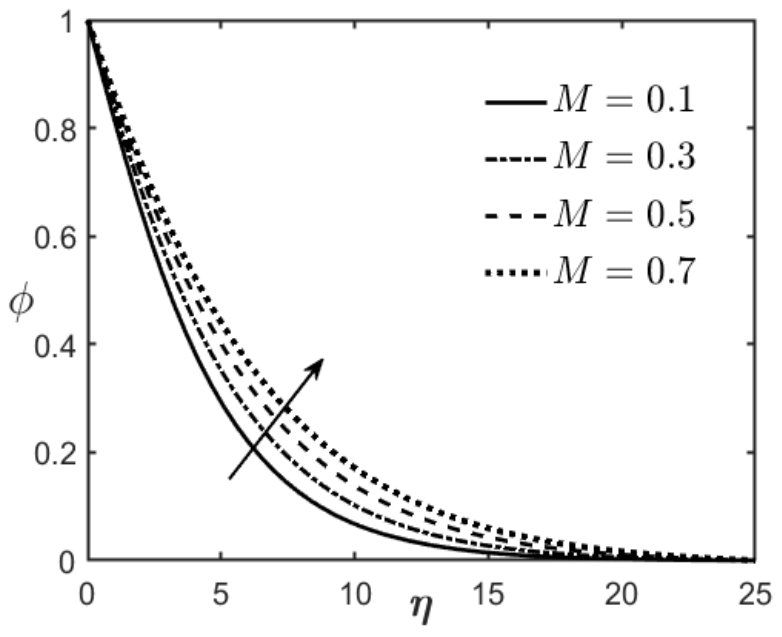

Fig. 11: Variation of Concentration against $\eta$ for various values of $M$.

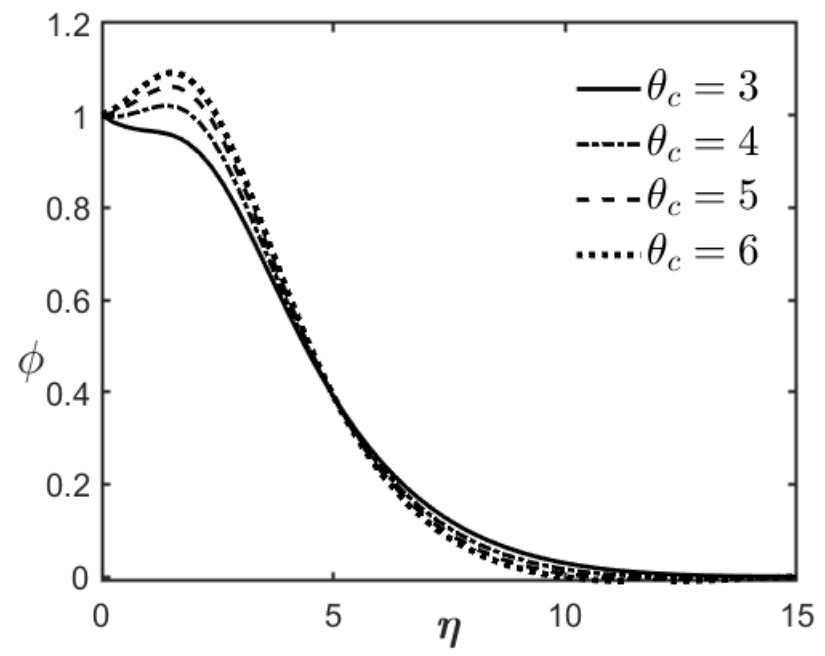

Fig. 12: Variation of Concentration against $\eta$ for various values of $\boldsymbol{\theta}_{\boldsymbol{c}}$.

Figs. $11-15$ present the variation of magnetic parameter $(M)$, thermal conductivity $\left(\boldsymbol{\theta}_{\boldsymbol{c}}\right)$, viscosity $\left(\boldsymbol{\theta}_{\boldsymbol{r}}\right)$, thermal stratification parameter $(S)$ and Schmidt number $(S c)$ on Concentration of Species. In Fig. 11, concentration increases with the rise in magnetic parameter $(M)$ and this effect is partially involved with concentration. 
Increasing the magnetic parameter increases the Lorentz force, which reduces the fluid velocity, and as a result, the concentration of the fluid increases. The impact of thermal conductivity $\left(\boldsymbol{\theta}_{\boldsymbol{c}}\right)$ on the concentration of the fluid is displayed in Fig. 12. This elucidates that concentration profile and its associated boundary layer thickness are increased with an increase in the thermal conductivity. Temperature decreases as parameter of thermal conductivity rises and as a result, the fluid concentration enhanced in fact a reversed behaviour is observed towards free stream.

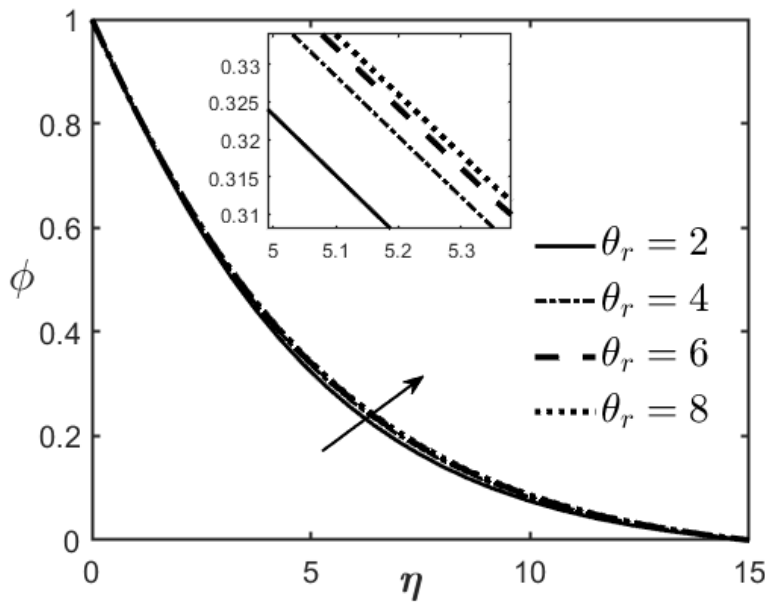

Fig. 13: Variation of Concentration against $\eta$ for various values of $\boldsymbol{\theta}_{\boldsymbol{r}}$.

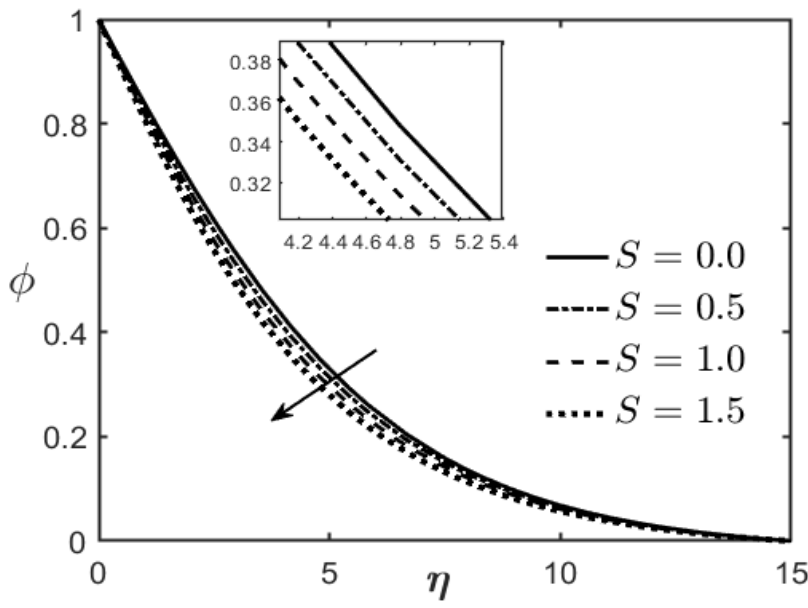

Fig. 14: Variation of Concentration against $\eta$ for various values of $S$.

In Fig. 13, the variation of viscosity $\left(\boldsymbol{\theta}_{\boldsymbol{r}}\right)$ has studied on concentration of species. As viscosity always retards the movement of molecules of fluid and it is not directly dependent on concentration and hence the concentration of species rises with the rise in viscosity parameter. In Fig. 14, the impact of thermal stratification parameter $(S)$ on the concentration profile is examined. From this Fig. 14 it is observed that the concentration profile and its related boundary layer thickness is declined monotonically with an increase in the thermal stratification parameter. However prescribed surface concentration case can be achieved when $S=0$ is used.

Fig.15 displays that the concentration profile for the impact of Schmidt number $(S c)$. An increase in $S c$ will suppress concentration in the boundary layer regime. It is a dimensionless number, which describe the property of the fluids that higher $S c$ will imply a decrease of molecular diffusivity causing a reduction in concentration boundary layer thickness. Lower Sc will result in higher concentrations i.e. greater molecular (species) diffusivity causing an increase in concentration boundary layer thickness.

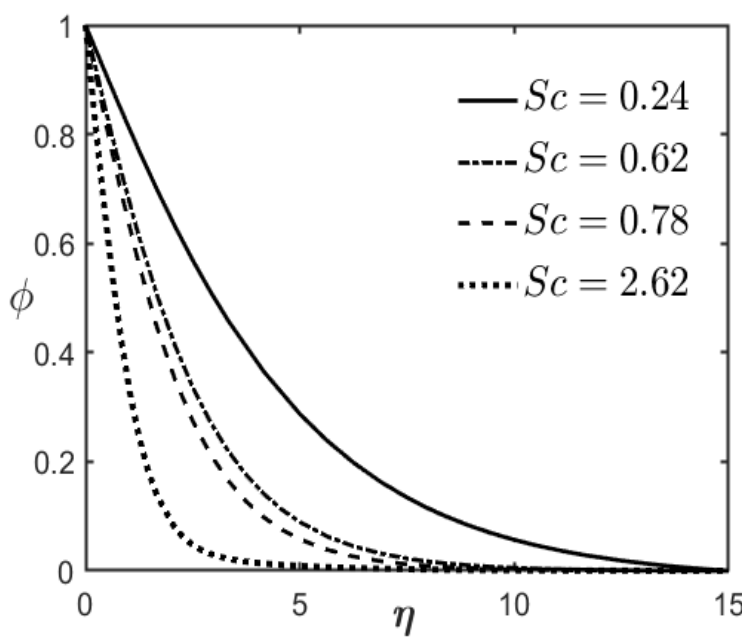

Fig. 15: Variation of Concentration against $\eta$ for various values of $S c$.

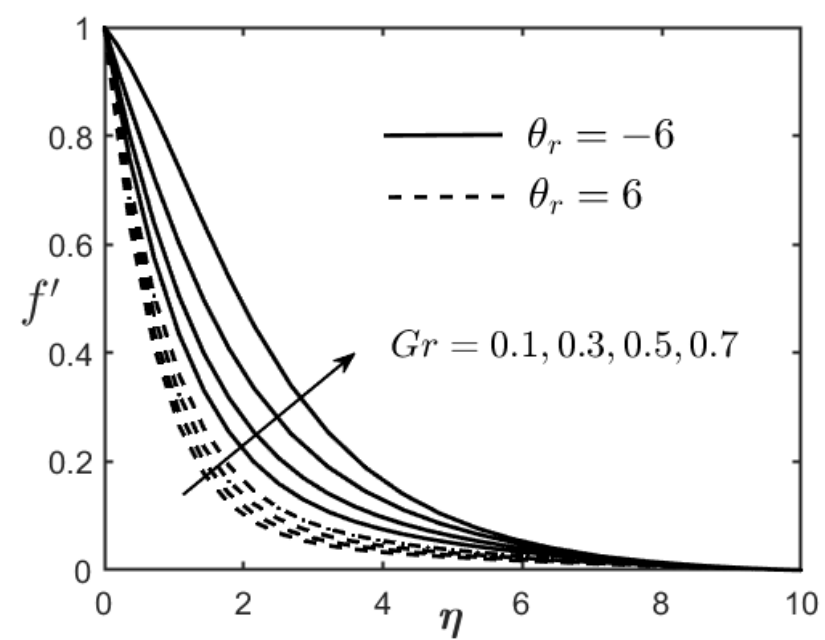

Fig. 16: Variation of Velocity against $\eta$ for various values of $\mathrm{Gr}$ and $\theta_{r}$. 
The impact of thermal buoyancy parameter $(G r)$ and viscosity $\left(\theta_{r}\right)$ on the fluid velocity $\left(f^{\prime}\right)$, temperature $(\theta)$ and concentration of species $(\phi)$ respectively presented in Figs. 16, 17 and 18. Positive Gr corresponds to cooling of the plate by free convection currents. $G r$ is proportional to the thermal buoyancy generated by free convection currents in the regime. Increasing buoyancy will therefore aid the flow. A positive increase in $\mathrm{Gr}$ from 0.1 , through $0.3,0.5$ to $0.7(G r<1)$ designates that viscous hydrodynamic forces exceed thermal buoyancy forces in the boundary layer), clearly accentuates velocities (Fig. 16) i.e. accelerates the flow. This pattern is sustained for some distance into the boundary layer, transverse to the plate. The velocity boundary layer thickness is shrunk due to the inertial forces between the molecules for $G r=0.1$ when $\theta_{r}>0$ (resistive forces). Conversely there is a consistent suppression of temperatures (Fig. 17) and concentrations (Fig. 18) with a rise in $G r$, as observed in Fig. 17 and 18. The maximum temperatures and concentrations have been detected corresponding to $G r=0.1$ for $\theta_{r}>0$ (resistive forces). Moreover, viscosity $\left(\theta_{r}>0\right)$ generates resistive forces which resists the motion of fluid molecules and $\theta_{r}<0$ represents no viscosity that means no resistance forces and therefore, fluid molecules are moving freely within the boundary layer. Thus the fluid velocity $\left(f^{\prime}\right)$ transited from no viscosity to viscosity. However, this behaviour is opposite to the thermal and concentration boundary layers.

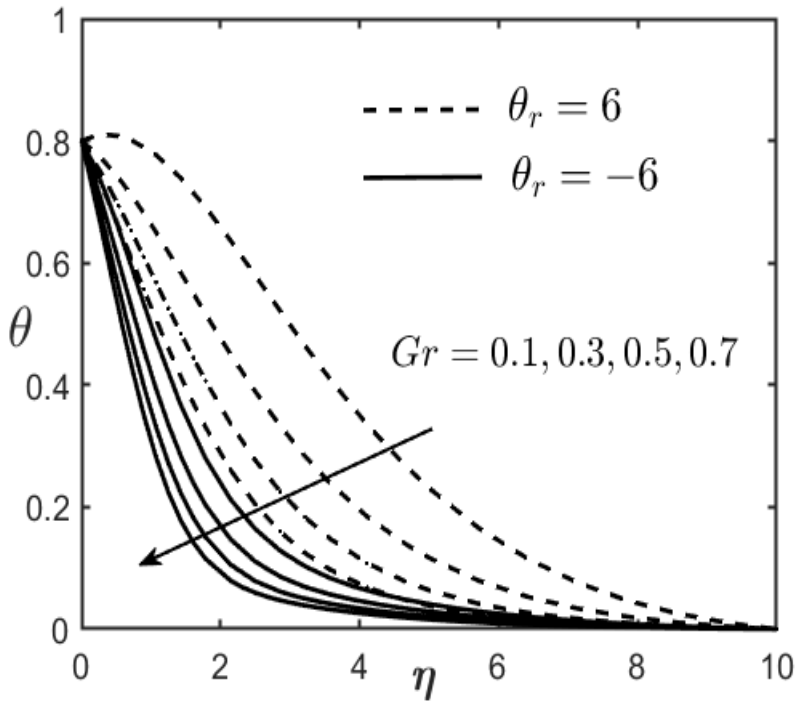

Fig. 17: Variation of temperature against $\eta$ for various values of $G r$ and $\theta_{r}$.

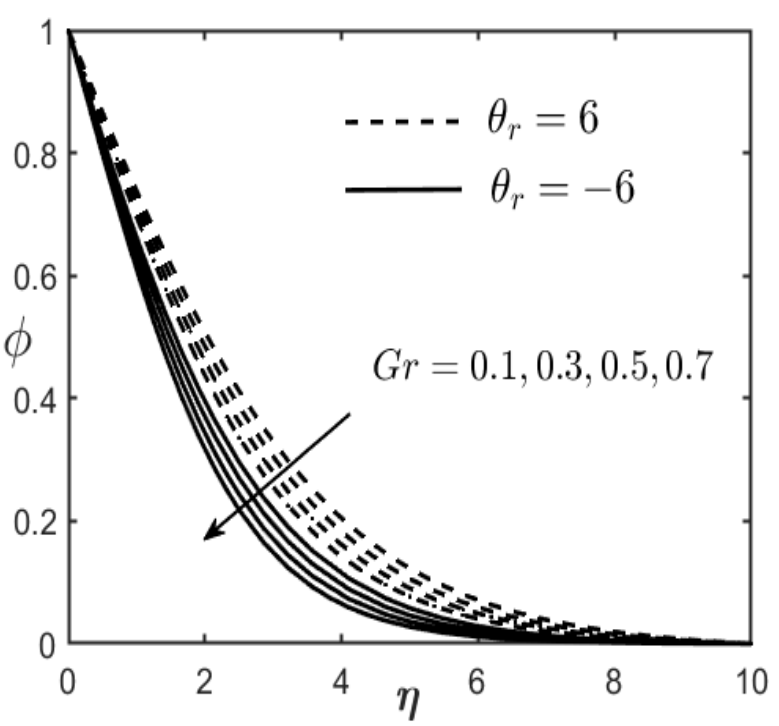

Fig. 18: Variation of Concentration against $\eta$ for various values of $G r$ and $\theta_{r}$.

Tables $4-9$ depict the results of $C_{f}, N u$ and $S h$ for various combination of parameters as mentioned. These tables give effects of viscosity and thermal conductivity over other parameters and have significant out comes. Using the above analysis, the effect of various parameters on $C_{f}, N u$ and $S h$ are shown in the following Tables when $M=0.5, \operatorname{Pr}=0.71, S c=0.24, G r=0.1=G c, S=0.2, D u=S r=Q=0.1, \theta_{r}=-8, \theta_{c}=-6$.

Table 4: Variation of $M$ and $\theta_{r}$ on $C_{f}, N u$ and $S h$.

\begin{tabular}{|c|c|c|c|c|r|r|r|c|c|}
\hline$M$ & \multicolumn{3}{|c|}{$\mathbf{0 . 1}$} & \multicolumn{3}{c|}{$\mathbf{1 . 0}$} & \multicolumn{3}{|c|}{$\mathbf{2 . 0}$} \\
\hline $\boldsymbol{\theta}_{\boldsymbol{r}}$ & $\boldsymbol{C}_{\boldsymbol{f}}$ & $\boldsymbol{N u}$ & $\boldsymbol{S h}$ & $\boldsymbol{C}_{\boldsymbol{f}}$ & $\boldsymbol{N u}$ & $\boldsymbol{S h}$ & $\boldsymbol{C}_{\boldsymbol{f}}$ & $\boldsymbol{N u}$ & $\boldsymbol{S h}$ \\
\hline-8 & 0.29094 & 0.20966 & 0.23582 & 0.89097 & 0.13276 & 0.21196 & 1.28547 & 0.09730 & 0.20260 \\
\hline-6 & 0.28460 & 0.20885 & 0.23553 & 0.87282 & 0.13144 & 0.21160 & 1.25886 & 0.09604 & 0.20227 \\
\hline-4 & 0.27286 & 0.20729 & 0.23498 & 0.83942 & 0.12892 & 0.21091 & 1.21003 & 0.09363 & 0.20164 \\
\hline-2 & 0.24352 & 0.20300 & 0.23347 & 0.75738 & 0.1222 & 0.20909 & 1.09078 & 0.08731 & 0.19999 \\
\hline 2 & 0.46653 & 0.22452 & 0.24131 & 1.41995 & 0.15913 & 0.21945 & 2.07849 & 0.12413 & 0.20970 \\
\hline 4 & 0.36938 & 0.21787 & 0.23881 & 1.12224 & 0.14681 & 0.2159 & 1.62849 & 0.11123 & 0.20626 \\
\hline 6 & 0.34749 & 0.21588 & 0.23808 & 1.05663 & 0.1433 & 0.21490 & 1.53048 & 0.10768 & 0.20532 \\
\hline 8 & 0.33774 & 0.21493 & 0.23773 & 1.02764 & 0.14163 & 0.21443 & 1.48733 & 0.10601 & 0.20488 \\
\hline
\end{tabular}


Table 5: Variation of $M$ and $\theta_{c}$ on $C_{f}, N u$ and $S h$

\begin{tabular}{|c|c|c|c|c|c|c|c|c|c|}
\hline$M$ & \multicolumn{4}{|c|}{$\mathbf{0 . 1}$} & \multicolumn{3}{|c|}{$\mathbf{1 . 0}$} & \multicolumn{2}{|c|}{$\mathbf{2 . 0}$} \\
\hline $\boldsymbol{\theta}_{\boldsymbol{c}}$ & $\boldsymbol{C}_{\boldsymbol{f}}$ & $\boldsymbol{N} \boldsymbol{u}$ & $\boldsymbol{S h}$ & $\boldsymbol{C}_{\boldsymbol{f}}$ & $\boldsymbol{N u}$ & $\boldsymbol{S h}$ & $\boldsymbol{C}_{f}$ & $\boldsymbol{N u}$ & $\boldsymbol{S h}$ \\
\hline-6 & 0.29094 & 0.20966 & 0.23582 & 0.89097 & 0.13275 & 0.211969 & 1.28546 & 0.09730 & 0.202603 \\
\hline-4 & 0.29104 & 0.20437 & 0.23558 & 0.89112 & 0.12943 & 0.211806 & 1.28557 & 0.09439 & 0.202493 \\
\hline-2 & 0.29133 & 0.19134 & 0.23487 & 0.89153 & 0.12146 & 0.211324 & 1.28590 & 0.08750 & 0.202170 \\
\hline-1 & 0.29182 & 0.17302 & 0.23355 & 0.89228 & 0.11083 & 0.210398 & 1.28654 & 0.07870 & 0.201539 \\
\hline 3 & 0.29022 & 0.26223 & 0.23738 & 0.89004 & 0.16776 & 0.213001 & 1.28475 & 0.12856 & 0.203293 \\
\hline 4 & 0.29035 & 0.24943 & 0.23710 & 0.89021 & 0.15899 & 0.212822 & 1.28489 & 0.12068 & 0.203172 \\
\hline 5 & 0.29043 & 0.24287 & 0.23694 & 0.89031 & 0.15454 & 0.212716 & 1.28496 & 0.11669 & 0.203101 \\
\hline 7 & 0.29052 & 0.23617 & 0.23676 & 0.89041 & 0.15004 & 0.212596 & 1.28503 & 0.11266 & 0.203021 \\
\hline
\end{tabular}

Table 6: Variation of $S c$ and $\theta_{r}$ on $C_{f}, N u$ and $S h$.

\begin{tabular}{|c|c|c|c|c|c|c|c|c|c|}
\hline$S c$ & \multicolumn{3}{|c|}{$\mathbf{0 . 2 4}$} & \multicolumn{3}{c|}{$\mathbf{0 . 6 2}$} & \multicolumn{3}{|c|}{$\mathbf{0 . 7 8}$} \\
\hline $\boldsymbol{\theta}_{\boldsymbol{r}}$ & $\boldsymbol{C}_{\boldsymbol{f}}$ & $\boldsymbol{N u}$ & $\boldsymbol{S h}$ & $\boldsymbol{C}_{\boldsymbol{f}}$ & $\boldsymbol{N u}$ & $\boldsymbol{S h}$ & $\boldsymbol{C}_{\boldsymbol{f}}$ & $\boldsymbol{N u}$ & $\boldsymbol{S h}$ \\
\hline-8 & 0.613619 & 0.165860 & 0.221458 & 0.62432 & 0.15381 & 0.31344 & 0.62830 & 0.14921 & 0.35227 \\
\hline-6 & 0.601229 & 0.164667 & 0.221099 & 0.61157 & 0.15262 & 0.31248 & 0.61542 & 0.14801 & 0.35108 \\
\hline-4 & 0.578372 & 0.162375 & 0.220412 & 0.58806 & 0.15032 & 0.31064 & 0.59168 & 0.14570 & 0.34881 \\
\hline-2 & 0.521844 & 0.156167 & 0.218576 & 0.52997 & 0.14412 & 0.30571 & 0.53305 & 0.13947 & 0.34269 \\
\hline 2 & 0.967413 & 0.188706 & 0.228613 & 0.98931 & 0.17691 & 0.33234 & 0.99715 & 0.17253 & 0.37544 \\
\hline 4 & 0.769652 & 0.178279 & 0.225280 & 0.78514 & 0.16633 & 0.32358 & 0.79080 & 0.16183 & 0.36475 \\
\hline 6 & 0.725679 & 0.175226 & 0.224326 & 0.73978 & 0.16324 & 0.32106 & 0.74495 & 0.15872 & 0.36166 \\
\hline 8 & 0.706185 & 0.173768 & 0.223874 & 0.71968 & 0.16177 & 0.31986 & 0.72464 & 0.15723 & 0.36018 \\
\hline
\end{tabular}

Table 7: Variation of $S c$ and $\theta_{c}$ on $C_{f}, N u$ and $S h$.

\begin{tabular}{|c|c|c|c|c|c|c|c|c|c|}
\hline$S c$ & \multicolumn{3}{|c|}{$\mathbf{0 . 2 4}$} & \multicolumn{3}{c|}{$\mathbf{0 . 6 2}$} & \multicolumn{3}{|c|}{$\mathbf{0 . 7 8}$} \\
\hline $\boldsymbol{\theta}_{\boldsymbol{c}}$ & $\boldsymbol{C}_{\boldsymbol{f}}$ & $\boldsymbol{N u}$ & $\boldsymbol{S h}$ & $\boldsymbol{C}_{\boldsymbol{f}}$ & $\boldsymbol{N u}$ & $\boldsymbol{S h}$ & $\boldsymbol{C}_{\boldsymbol{f}}$ & $\boldsymbol{N u}$ & $\boldsymbol{S h}$ \\
\hline-6 & 0.61361 & 0.16586 & 0.22145 & 0.62432 & 0.15381 & 0.31344 & 0.62830 & 0.14921 & 0.35227 \\
\hline-4 & 0.61376 & 0.16184 & 0.22125 & 0.62443 & 0.15005 & 0.31297 & 0.62840 & 0.14554 & 0.35171 \\
\hline-2 & 0.61415 & 0.15204 & 0.22066 & 0.62476 & 0.14089 & 0.31161 & 0.62870 & 0.13661 & 0.35008 \\
\hline-1 & 0.61487 & 0.13855 & 0.21953 & 0.62535 & 0.12832 & 0.30903 & 0.62926 & 0.12439 & 0.34699 \\
\hline 3 & 0.61267 & 0.20695 & 0.22274 & 0.62358 & 0.19253 & 0.31643 & 0.62763 & 0.18705 & 0.35587 \\
\hline 4 & 0.61284 & 0.19679 & 0.22252 & 0.62371 & 0.18293 & 0.31591 & 0.62775 & 0.17766 & 0.35523 \\
\hline 5 & 0.61294 & 0.19161 & 0.22239 & 0.62379 & 0.17805 & 0.31560 & 0.62782 & 0.17288 & 0.35486 \\
\hline 7 & 0.61305 & 0.18635 & 0.22224 & 0.62387 & 0.17309 & 0.31525 & 0.62790 & 0.16804 & 0.35444 \\
\hline
\end{tabular}

Table 8: Variation of $S$ and $\theta_{r}$ on $C_{f}, N u$ and $S h$.

\begin{tabular}{|c|c|c|c|c|c|c|c|c|c|}
\hline$S$ & \multicolumn{3}{c|}{$\mathbf{0 . 0}$} & \multicolumn{3}{c|}{$\mathbf{0 . 5}$} & \multicolumn{1}{|c|}{$\mathbf{1 . 0}$} \\
\hline $\boldsymbol{\theta}_{\boldsymbol{r}}$ & $\boldsymbol{C}_{\boldsymbol{f}}$ & $\boldsymbol{N u}$ & $\boldsymbol{S h}$ & $\boldsymbol{C}_{\boldsymbol{f}}$ & $\boldsymbol{N u}$ & $\boldsymbol{S h}$ & $\boldsymbol{C}_{\boldsymbol{f}}$ & $\boldsymbol{N u}$ & $\boldsymbol{S h}$ \\
\hline-8 & 0.62109 & 0.21233 & 0.22064 & 0.60223 & 0.09894 & 0.22261 & 0.58278 & -0.00486 & 0.22436 \\
\hline-6 & 0.61063 & 0.21046 & 0.22022 & 0.58682 & 0.09848 & 0.22236 & 0.56193 & -0.00486 & 0.22436 \\
\hline-4 & 0.59098 & 0.20690 & 0.21942 & 0.55883 & 0.09758 & 0.22189 & 0.52440 & -0.00486 & 0.22436 \\
\hline-2 & 0.54052 & 0.19747 & 0.21732 & 0.49212 & 0.09506 & 0.22057 & 0.43682 & -0.00486 & 0.22435 \\
\hline 2 & 0.85633 & 0.25041 & 0.22978 & 1.11258 & 0.10700 & 0.22696 & 1.31256 & -0.00486 & 0.22438 \\
\hline 4 & 0.74013 & 0.23247 & 0.22536 & 0.81132 & 0.10349 & 0.22504 & 0.87469 & -0.00486 & 0.22438 \\
\hline 6 & 0.70904 & 0.22740 & 0.22415 & 0.74963 & 0.10241 & 0.22446 & 0.78712 & -0.00486 & 0.22437 \\
\hline 8 & 0.69463 & 0.22500 & 0.22358 & 0.72297 & 0.10189 & 0.22418 & 0.74959 & -0.00486 & 0.22437 \\
\hline
\end{tabular}


Table 9: Variation of $S$ and $\theta_{c}$ on $C_{f}, N u$ and $S h$.

\begin{tabular}{|c|c|c|c|c|c|c|c|c|c|}
\hline$S$ & \multicolumn{3}{|c|}{$\mathbf{0 . 0}$} & \multicolumn{3}{c|}{$\mathbf{0 . 5}$} & \multicolumn{1}{|c|}{$\mathbf{~ . 0}$} \\
\hline $\boldsymbol{\theta}_{\boldsymbol{c}}$ & $\boldsymbol{C}_{\boldsymbol{f}}$ & $\boldsymbol{N u}$ & $\boldsymbol{S h}$ & $\boldsymbol{C}_{\boldsymbol{f}}$ & $\boldsymbol{N u}$ & $\boldsymbol{S h}$ & $\boldsymbol{C}_{f}$ & $\boldsymbol{N u}$ & $\boldsymbol{S h}$ \\
\hline-6 & 0.62109 & 0.21233 & 0.22064 & 0.60223 & 0.09894 & 0.22261 & 0.58278 & -0.00486 & 0.22436 \\
\hline-4 & 0.62130 & 0.20913 & 0.22033 & 0.60229 & 0.09508 & 0.22253 & 0.58278 & -0.00453 & 0.22436 \\
\hline-2 & 0.62190 & 0.20088 & 0.21941 & 0.60245 & 0.08596 & 0.22230 & 0.58278 & -0.00378 & 0.22436 \\
\hline-1 & 0.62190 & 0.20088 & 0.21941 & 0.60245 & 0.08596 & 0.22230 & 0.58278 & -0.00378 & 0.22436 \\
\hline 3 & 0.61962 & 0.24042 & 0.22268 & 0.60187 & 0.14136 & 0.22310 & 0.58278 & -0.00850 & 0.22436 \\
\hline 4 & 0.61989 & 0.23413 & 0.22232 & 0.60193 & 0.13047 & 0.22301 & 0.58278 & -0.00756 & 0.22436 \\
\hline 5 & 0.62005 & 0.23078 & 0.22211 & 0.60197 & 0.12501 & 0.22296 & 0.58278 & -0.00709 & 0.22436 \\
\hline 7 & 0.62022 & 0.22726 & 0.22187 & 0.60201 & 0.11953 & 0.22291 & 0.58278 & -0.00661 & 0.22436 \\
\hline
\end{tabular}

In Table 4, increasing $M$ and $\theta_{c}$ leads to decrease $N u$ and $S h$, but to increase $C_{f}$. A similar behaviour is observed in Table 5 also. Through Table 6, increasing $S c$ and $\boldsymbol{\theta}_{c}$ raises the values of $C_{f}$ and $S h$, but reduces $\mathrm{Nu}$, and similar effects are seen in Table 7. In Table 8 and $9, C_{f}$ and $N u$ decreases for increasing $S, \boldsymbol{\theta}_{\boldsymbol{r}}$ and $\boldsymbol{\theta}_{\boldsymbol{c}}$, but increases for increase in $S h$.

\section{Conclusions}

The relevance study of the problem under discussion is revealed from the above discussion. From the above analysis, we may say that for accurate calculations of the velocity, temperature and concentration, variable property of viscosity and thermal conductivity should be considered. The whole analysis can be concluded as below:

1. Increase in the values of magnetic parameter and viscosity parameter, both temperature and concentration increase but velocity decreases.

2. The fluid temperature decreases with the thermal conductivity parameter and stratification parameter.

3. Fluid velocity and concentration rise with the rise in thermal conductivity parameter and stratification parameter.

4. Both velocity and concentration decline but temperature gets higher with the rise of Schmidt number.

5. Enhancement of viscosity parameter, skin friction coefficient rises but both Nusselt number and Sherwood number decline.

6. The coefficient of skin friction increase and both Nusselt number and Sherwood number decrease with the rise in thermal conductivity parameter.

7. Effects of the viscosity and thermal conductivity parameters are prominent. Hence to compute accurately the velocity, temperature and concentration profile, these properties should be treated as variable.

\section{References}

Cheesewright, R. (1967): Natural Convection from a plate, vertical surface in non-isothermal surroundings, Int. J. Heat Mass Trans., Vol.10, pp.1847-1859. https://doi.org/10.1016/0017-9310(67)90054-3

Helmy, K.A. (1998): MHD unsteady free convection flow past a vertical porous plate, ZAMM, Vol.78, pp. 255270. https://dx.doi.org/10.1002/(SICI)1521-4001(199804)78

Pop, I. and Soundalgekar, V. M. (1962): Viscous dissipation effects on unsteady free convective flow past an infinite vertical porous plate with variable suction, Int. J. Heat Mass Transfer, Vol.17, No. 1, pp. 85-92. https://doi.org/10.1016/0017-9310(74)90041-6

Molla, M. M., Hossain, M. A. and Yao, L. S. (2004): Radiation effect on mixed convection laminar flow along a vertical wavy surface, Int. Journal of Thermal Science, Vol. 43, pp.157-163. https://dx.doi.org/10.1016/j.ijthermalsci.2006.10.010 
Mahanti, N.C. and Gaur, P. (2009): Effect of varying viscosity and thermal conductivity on steady free convective flow and heat transfer along an isothermal vertical plate in the presence of heat sink, J. Appl. Fluid Mechanics, Vol. 2. No. 1, pp. 23-28. https://doi.org/10.36884/jafm.2.01.11852

Hazarika, G.C., Joydeep, B. and Jadav, K. (2018): Effects of variable viscosity and thermal conductivity on hydromagnetic transient flow over a hot vertical plate, Int. Journal of Scientific Research, Vol. 7, No. 7, pp. 543-555.

Sarma, U. and Hazarika, G.C. (2009): Effect of variable viscosity and thermal conductivity on heat and mass transfer flow along a vertical plate in the presence of magnetic field, Int. J. Phy. Education, Vol. 8, No. 3.

Thakur, P.M., Hazarika, G. C. (2015): Effects of variable viscosity and thermal conductivity on the MHD flow of micropolar fluid past an accelerated infinite vertical insulated plate, Int. J. of Heat and Tech. USA., Vol. 33, pp. 73-78. https://doi.org/10.18280/ijht.330310

Lai F.C. and Kulacki, F.A. (1990): The effect of variable viscosity on convective heat and mass transfer along a vertical surface in saturated porous medium, Int. J. Heat Mass Tran., Vol. 33, No. 5, 1028-1031. https://dx.doi.org/10.1016/0017-9310(90)90084-S

Hazarika, G.C., Phukan, B., and Ahmed, S. (2020): The effect of variable viscosity and thermal conductivity on unsteady free convective flow of a micropolar fluid past a vertical cone, Journal of Engineering Physics and Thermophysics, Vol. 93, pp. 184-191. https://doi.org/10.1007/s10891-020-02106-7

Subhakar, M. J., Gangadhar, K. and Reddy, N. B. (2012): Soret and Dufour effects on MHD convective flow of heat and mass transfer over a moving non-isothermal vertical plate with heat generation/absorption, Advances in Applied Science Research, Vol. 3, No. 5, pp. 3165-3184.

Herwig, H. and Gersten, K. (1986): The effect of variable properties on laminar boundary layer flow, Warme Stoffubertr., Vol. 20, 47. https://doi.org/10.1007/BF00999737

Kalita, K. and Ahmed, S, (2016): Laplace transformation for optically thick gray gas in a vertical channel with magnetic body force: a Rosseland approximation, J. of Engineering Physics and Thermophysics, Vol. 90, No. 4, pp. 918-931. https://dx.doi.org:10.1007/s10891-017-1639-1

Zueco, J., Ahmed, S. and López-González, L. M. (2017): 2-D Unsteady free convective heat and mass transfer Newtonian Hartmann flow with thermal diffusion and Soret effects: Network model and finite differences, Int. Journal of Heat and Mass Transfer, Vol. 110, pp. 467-475.

https://doi.org/10.1016/j.ijheatmasstransfer.2017.03.046

Ahmed, S., Zueco, J. and López-González, L. M. (2017): Numerical and analytical solutions for magnetohydrodynamic 3D flow through two parallel porous plates, Int. Journal of Heat and Mass Transfer, Vol. 108, pp. 322-331. https://dx.doi.org/10.1016/j.ijheatmasstransfer.2016.11.102

https://doi.org/10.1016/j.ijheatmasstransfer.2016.11.102

Kalita D, Hazarika S, Ahmed S (2020) Applications of CNTs in a vertical channel of porous medium for human blood flow: a rheological model. JP Journal of Heat and Mass Transfer 20 (2): 105-120.

https://doi.org/10.17654/HM020020105

Hazarika, S. and Ahmed, S. (2021): Study of Carbon Nanotubes with Casson Fluid in a Vertical Channel of Porous Media for Hydromagnetic Drag Force and Diffusion-Thermo, J. Sci. Res., Vol. 13, No. 1.

Khedr, M.-E. M., Chamkha, A. J. and Bayomi, M. (2009): MHD flow of a micropolar fluid past a stretched permeable surface with heat generation or absorption, Nonlinear Analysis: Modeling and Control, Vol. 14, No. 1, pp. 27-40. https://doi.org/10.15388/NA.2009.14.1.14528

Modather, M., Rashad, A. M. and Chamkha, A. J. (2009): An analytical study of MHD heat and mass transfer oscillatory flow of a micropolar fluid over a vertical permeable plate in a porous medium, Turkish J. Eng. Env. Sci. 33 (2009), 245 - 257. https://doi:10.3906/muh-0906-31

Magyari, E. and Chamkha, Ali J. (2010): Combined effect of heat generation or absorption and first-order chemical reaction on micropolar fluid flows over a uniformly stretched permeable surface: The full analytical solution, Int. Journal of Thermal Sciences, Vol. 49, No. 9, pp. 1821-1828.

https://doi.org/10.1016/j.ijthermalsci.2010.04.007

Takhar, H.S., Chamkha, A.J. and Nath, G. (1999): Unsteady flow and heat transfer on a semi-infinite flat plate with an aligned magnetic field, Int. Journal of Engineering Science, Int. J. Engineering Science, Vol. 37, No. 13, pp. 1723-1736. https://doi.org/10.1016/S0020-7225(98)00144-X

Chamkha, A.J. and Aly A.M. (2010): MHD free convection flow of a nanofluid past a vertical plate in the presence of heat generation or absorption effects Vol. 198, No. 3, pp. 425-441.

https://doi.org/10.1080/00986445.2010.520232

Parvin, S., Nasrin, R., Alim, M.A., Hossain, N.F. and Chamkha, Ali J. (2012): Thermal conductivity variation on natural convection flow of water-alumina nanofluid in an annulus, Int. Journal of Heat and Mass Transfer, Vol. 55, No. 19-20, pp. 5268-5274. https://doi.org/10.1016/j.ijheatmasstransfer.2012.05.035 